\title{
Materialflusskostenrechnung - die internationale Karriere einer Methode zu Identifikation von Ineffizienzen in Produktionssystemen
}

\author{
Bernd Wagner $\cdot$ Michiasu Nakajima $\cdot$ Martina Prox
}

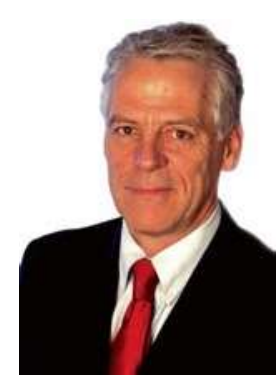

Bernd Wagner

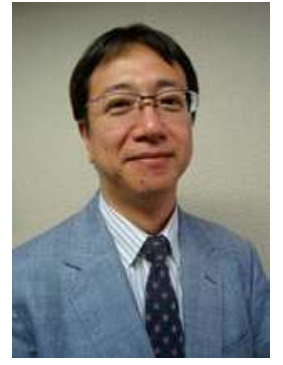

Michiasu Nakajima

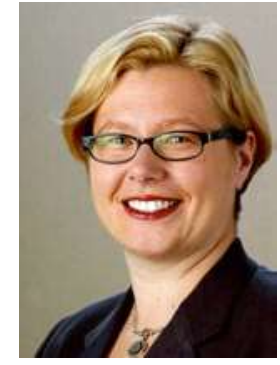

Martina Prox

\section{Entstehung und Entwicklung}

Die Materialflusskostenrechnung gehört zu den materialund energieflussorientierten Kostenrechnungsansätzen, die zur der Steigerung der betrieblichen Material-, Öko- und Kosteneffizienz eingesetzt werden können. Material- und energieflussorientierte Kostenrechnungsansätze stellen, wie der Name sagt, die betrieblichen Material- und Energieflüsse ins Zentrum der Kostenbetrachtung. Grund hierfür ist die zweifache Bedeutung von Material- und Energieflüssen.

\section{Prof. Dr. B. Wagner ( $\square)$}

Wissenschaftszentrum Umwelt (WZU), Universität Augsburg, Universitätsstrasse 1a, 86159 Augsburg, Deutschland

E-Mail: wagner@wzu.uni-augsburg.de

\section{Prof. Dr. M. Nakajima}

Faculty of Commerce, Kansai Universität Osaka,

3-3-35 Yamate-cho, Suita, Osaka 564-8680, Japan

E-Mail: nakajima@kansai-u.ac.jp

\section{Prox}

ifu Hamburg GmbH, Max-Brauer-Allee 50,

22765 Hamburg, Deutschland

E-Mail: m.prox@ifu.com
Zum Einen stehen die meisten direkten Umwelteinwirkungen von Unternehmen in unmittelbarem Zusammenhang mit den Material- und Energieflüssen des Produktionssystems. Zum Anderen stellen, jedenfalls in produzierenden Unternehmen, Energie und insbesondere Material häufig den größten Kostenblock dar. Somit liegt in der Reduzierung des Material- und Energieeinsatzes das gemeinsame Ziel von wirtschaftlichen und ökologischen Interessen (vgl. Loew et al. 2003).

Die Anfänge der Methode ergaben sich Ende der 80er Jahre aus betrieblichen Ökobilanzen als Masse- bzw. InputOutput-Bilanzen (vgl. Wagner und Strobel 1999 sowie Wagner und Rauberger 1999). In frühen Praxisprojekten zum Umweltmanagement hatte sich gezeigt, dass Materialeffizienz nur optimiert werden kann, wenn die Prozesse oder Materialflüsse in der Blackbox der Unternehmensbilanz beherrscht werden. Doch Beherrschung setzt Transparenz von physikalischen Mengen und Kosten der Materialflüsse voraus. Die Flusskostenrechnung bemühte sich um eine Quantifizierung und Bewertung aller Material- und Energieströme eines definierten Produktionssystems und die Anbindung an die vorhandene Kostenrechnung.

Parallel dazu entwickelte sich Mitte der 1990er Jahre auch die so genannte „Reststoffkostenrechnung“. Die Reststoffkostenrechnung hatte zum Ziel, Transparenz hinsichtlich der in den Reststoffen der Produktion kumulierten Kosten zu schaffen. Reststoff- oder auch Abfallkosten beinhalten neben den eigentlichen Entsorgungskosten auch den Materialwert, der sich aus dem Preis der eingekauften Rohstoffe ergibt, sowie die Kosten für den im Material angehäuften Wertschöpfungsanteil, für die Lagerung, das Handling der Reststoffe und für Umweltschutz-Aktivitäten (sog. End-of-Pipe-Behandlungen). Gewöhnlich entfällt der größte Kostenanteil auf den Materialwert. Im Gegensatz zu konventionellen Kostenrechnungsansätzen, ordnet die Rest- 
stoffkostenrechnung den Reststoffen eines Produktionssystems einen Kostenanteil zu und weist so den Reststoff als weiteren Kostenträger aus (vgl. Loew et al. 2003).

Die Flusskostenrechnung wurde durch Pilotprojekte, u. a. bei der Ciba Geigy Pharma-Deutschland GmbH, ITT Automotive GmbH oder der Firmengruppe Merckle-Ratiopharm methodisch ausgearbeitet und in der Unternehmenspraxis erprobt. Diese Projekte wurden von der Deutschen Bundesstiftung Umwelt (DBU), dem Hessischen Wirtschaftsministerium sowie dem Baden-Württembergischen Umweltministerium gefördert. Die Dokumentation der Methodik der Flusskostenrechnung und eine Vorgehensweise zur praktischen Umsetzung finden sich in zwei Leitfäden (LFU Baden-Württemberg 1999; Hessisches Wirtschaftsministerium 1999). Die Methode, die genannten Pilotprojekte und auch die Leitfäden sind in erster Linie auf Arbeiten des Institut für Management und Umwelt (IMU) Augsburg zurückzuführen (Berger et al. 2003).

In zwei groß angelegten Forschungsprojekten ECO-Rapid und ECO-Effizienz wurde die Flusskostenrechnung methodisch weiterentwickelt und in ca. 15 weiteren Industrieunternehmen in unterschiedlichen Branchen umgesetzt. Aus diesen Projekten sind diverse deutsche Fallstudien erarbeitet worden, die das Potenzial der Methodik zur Identifizierung und Reduktion von Ineffizienzen aufzeigen konnten. Insgesamt stellt die Flusskostenrechnung einen umfassenden Ansatz dar, für dessen Umsetzung eine Integration in die betrieblichen Abläufe, sowie in die zugehörigen IT- bzw. Enterprise Ressource Planning (ERP)-Systeme angestrebt wird. Das Ziel der Vollständigkeit hinsichtlich der Transparenz aller Stoff- und Energieströme bei der Flusskostenrechnung, stellt nach wie vor eine große Herausforderung für die Implementierung dar.

Als Materialflusskostenrechnung (engl. Material Flow Cost Accounting $=$ MFCA) erhebt die Methode nicht notwendig den Anspruch der vollständigen Erfassung aller Material- und Energieflüsse und kann auch auf ausgewählte
Bereiche des Produktionssystems oder ausgewählte Materialflüsse angewendet werden. MFCA kombiniert physikalische und kostenbezogene Informationen mit dem Ziel, in einem Unternehmen Ineffizienzen und Verschwendung zu minimieren, damit die Ressourceneffizienz zu erhöhen und gleichzeitig Umweltleistungen $\mathrm{zu}$ verbessern (vgl. z. B. Jasch 2009; METI 2007; Strobel und Redmann 2002; Wagner und Enzler 2006). MFCA ist von ihrem Ansatz her im Prinzip auch mit anderen Konzepten wie Umwelt- oder Prozessmanagement, Prozesskostenrechnung, Materialflussanalyse (vgl. z. B. Brunner und Rechberger 2003) oder Umweltrechnungslegung (vgl. z. B. Schaltegger und Burritt 2000) kombinierbar.

Mittlerweile entstanden noch weitere ähnliche und ebenfalls umweltorientierte Kostenrechnungsansätze, wie z. B. die Ressourcenkostenrechnung (vgl. Letmathe et al. 2002), die in einem mehrstufigen Verfahren auch zu einer Verknüpfung von physischen Material- und Energieströmen mit Kosten führt.

\section{MFCA - der methodische Ansatz}

Die Quantifizierung von Materialflüssen, Materialineffizienzen, insbesondere Materialverlusten sowie anderen Prozessineffizienzen ist das übergeordnete Ziel der Materialflusskostenrechnung. Sie geht damit über den Anspruch einer reinen Reststoffkosten- oder Umweltschutzkostenrechnung hinaus und verfolgt neben Reststoffen auch Produktmaterial und neben Umweltschutzkosten auch andere materialflussbezogene Kostenarten.

Grundzüge des Ansatzes sind in Abb. 1 veranschaulicht. Wesentliche Unterschiede zur konventionellen Kostenrechnung liegen zum Einen darin, dass zusätzliche, meist am Ort von Materialverlusten orientierte Kostenstellen, sog. Mengen- oder Materialstellen, eingeführt werden. Zum Anderen werden den Materialflüssen sowohl ihre Einkaufskosten,

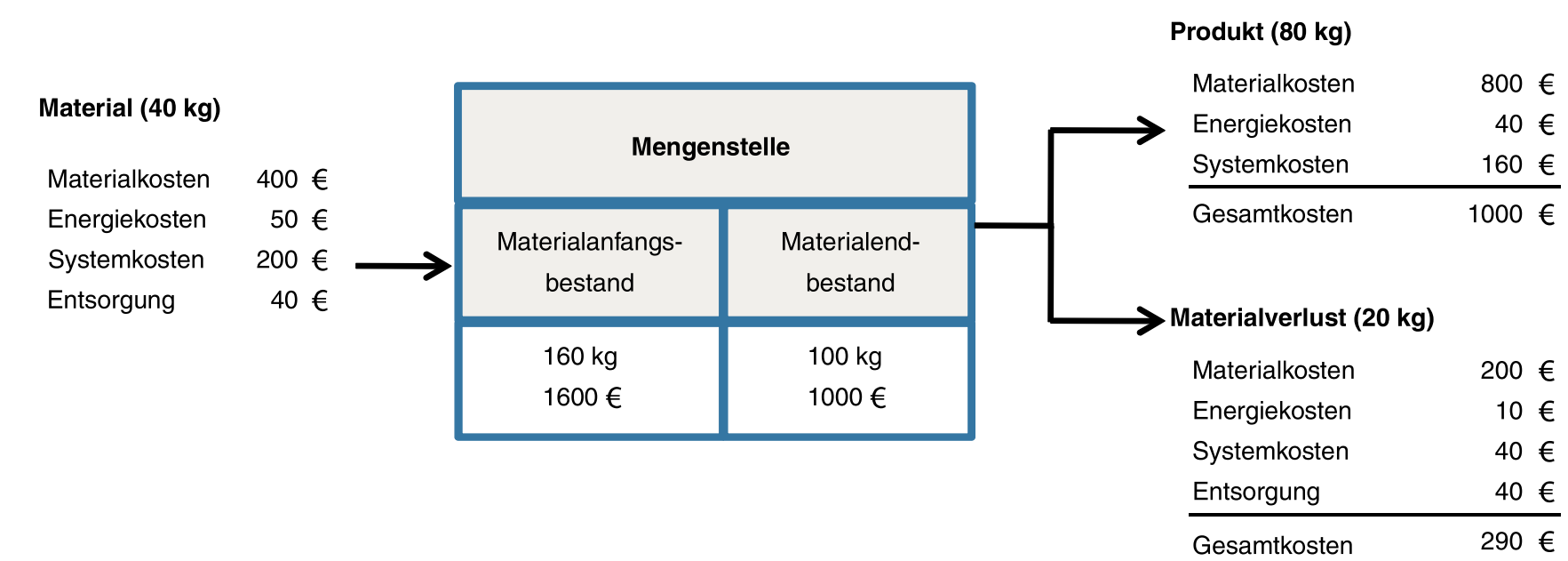

Abb. 1 Die Grundrechnung in der MFCA-Methodik. (In Anlehnung an DIN EN ISO 140512010 ) 
Tab. 1 Beispiel einer Materialflusskostenmatrix

\begin{tabular}{llllll}
\hline & $\begin{array}{l}\text { Material- } \\
\text { kosten } \\
(€)\end{array}$ & $\begin{array}{l}\text { Energie- } \\
\text { kosten } \\
(€)\end{array}$ & $\begin{array}{l}\text { System- } \\
\text { kosten } \\
(€)\end{array}$ & $\begin{array}{l}\text { Entsor- } \\
\text { gungs- } \\
\text { kosten }(€)\end{array}$ & $\begin{array}{l}\text { Gesamt- } \\
\text { kosten } \\
(€)\end{array}$ \\
\hline $\begin{array}{l}\text { Produkt } \\
\text { Materialverlust }\end{array}$ & 200 & 40 & 160 & & 1000 \\
$\begin{array}{l}\text { Entsorgung } \\
\text { Gesamt }\end{array}$ & 10 & 40 & & 290 \\
& 1000 & 50 & 200 & 40 & 1290 \\
\hline
\end{tabular}

wie auch die im Zuge des betrieblichen Wertschöpfungsprozesses akkumulierten Handling- und Systemkosten zugeordnet und transparent gehalten. Häufig werden die Kosten für MFCA als so genannte Materialflusskostenmatrix (engl. material flow cost matrix) dargestellt. Tabelle 1 zeigt die Kosten des einfachen Beispiels aus Abb. 1 in dieser Form.

Die Zurechnung (Allokation) der verschiedenen Kosten (Personal, Strom, Abschreibungen etc.) zu den einzelnen Flüssen bzw. Mengenstellen, erfolgt im einfachsten Fall nach Masse. MFCA geht dann von einem linearen Verhältnis zwischen Eingangs- und Ausgangsmaterial (Input und Output) eines Prozesses aus: Eine Reduktion von Produktionsabfällen führt daher entweder zu einer Reduktion der Inputflüsse oder zu einer Erhöhung des Produktoutputs.

Zum Beispiel würde eine Reduktion der Abfälle um $10 \mathrm{~kg}$ in Abb. 1 entweder, bei konstanter Produktionsmenge, den Materialbedarf und die damit verbundenen Kosten um $10 \%$ reduzieren. Das wären $100 €$; dazu kämen die Einsparungen bei den Entsorgungskosten von $20 €$, bei den Energiekosten von $5 €$ und bei den Systemkosten von $20 €$. Oder es könnte die Produktmenge bei konstantem Materialeinsatz um $10 \mathrm{~kg}$ gesteigert werden. Die Information, dass die Kosten durch die Materialverluste sich insgesamt auf $290 €$ summieren und nicht nur auf die Entsorgungskosten von $40 €$, unterstützt Manager oder andere Entscheidungsträger darin, gegen Ineffizienzen im Produktionsprozess vorzugehen.

Der Ansatz mit den Mengenstellen kann auf beliebig große Produktionssysteme übertragen werden. Abbildung 2 zeigt ein vereinfachtes zweistufiges Modell mit zwei Mengenstellen, bei dem die verschiedenen Kosten zwischen dem Produkt- und Reststoffstrom aufgeteilt werden. Hätte man den Reststoffstrom nur anhand seiner Entsorgungskosten bewertet, lägen seine Kosten bei $500 €$. Unter Einbeziehung der Materialkosten verursacht der Reststoffstrom immerhin schon $2800 €$. Einschließlich der System- und Energiekosten liegt das Einsparpotenzial schließlich bei $3815 €$. Dieser Betrag könnte im Gesamtsystem eingespart werden, wenn die Reststoffe gänzlich vermieden werden.

Die Leistungsfähigkeit der Materialflusskostenrechnung kann auch auf eine Wertschöpfungskette oder einen ganzen Produktlebenszyklus erweitert werden. Abbildung 3 zeigt ein vereinfachtes Lebenszyklusmodel, bei dem die Flüsse nach Produkt- und Reststoffanteil unterschieden sind und damit die möglichen Einsparpotenziale angedeutet werden. Da die Analyse auf Mengenangaben der eingesetzten Energien und Materialien basiert, kann sie nicht nur in Wertgrößen (Euro), sondern prinzipiell auch in ökologischen Wertgrößen (z. B. Treibhausgasemissionen) beziffert werden. Damit könnte man dann sogar die Umweltentlastung

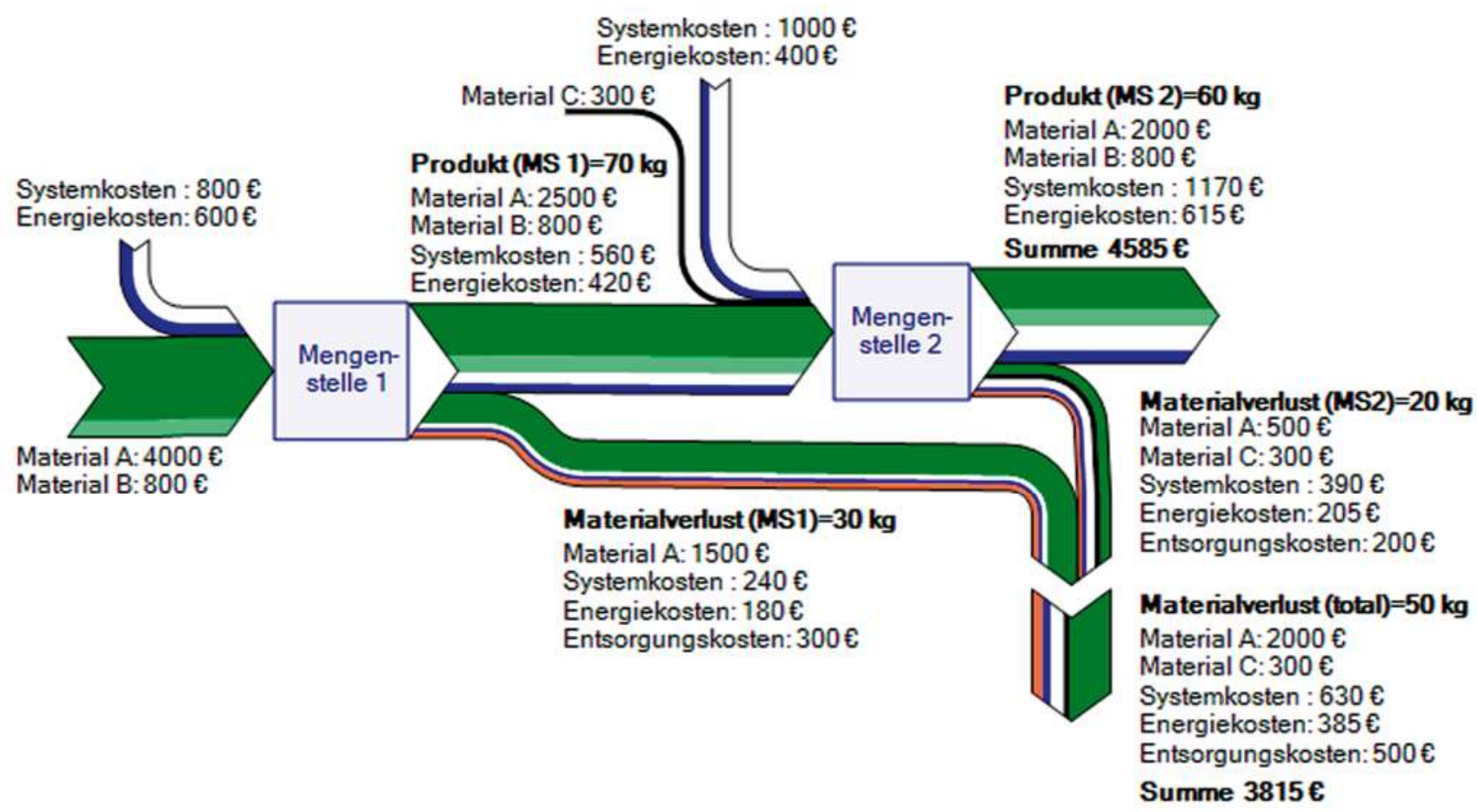

Abb. 2 Grafische Darstellung der Materialflusskosten mittels eines Sankey-Diagramms. (Viere und Möller 2009) 


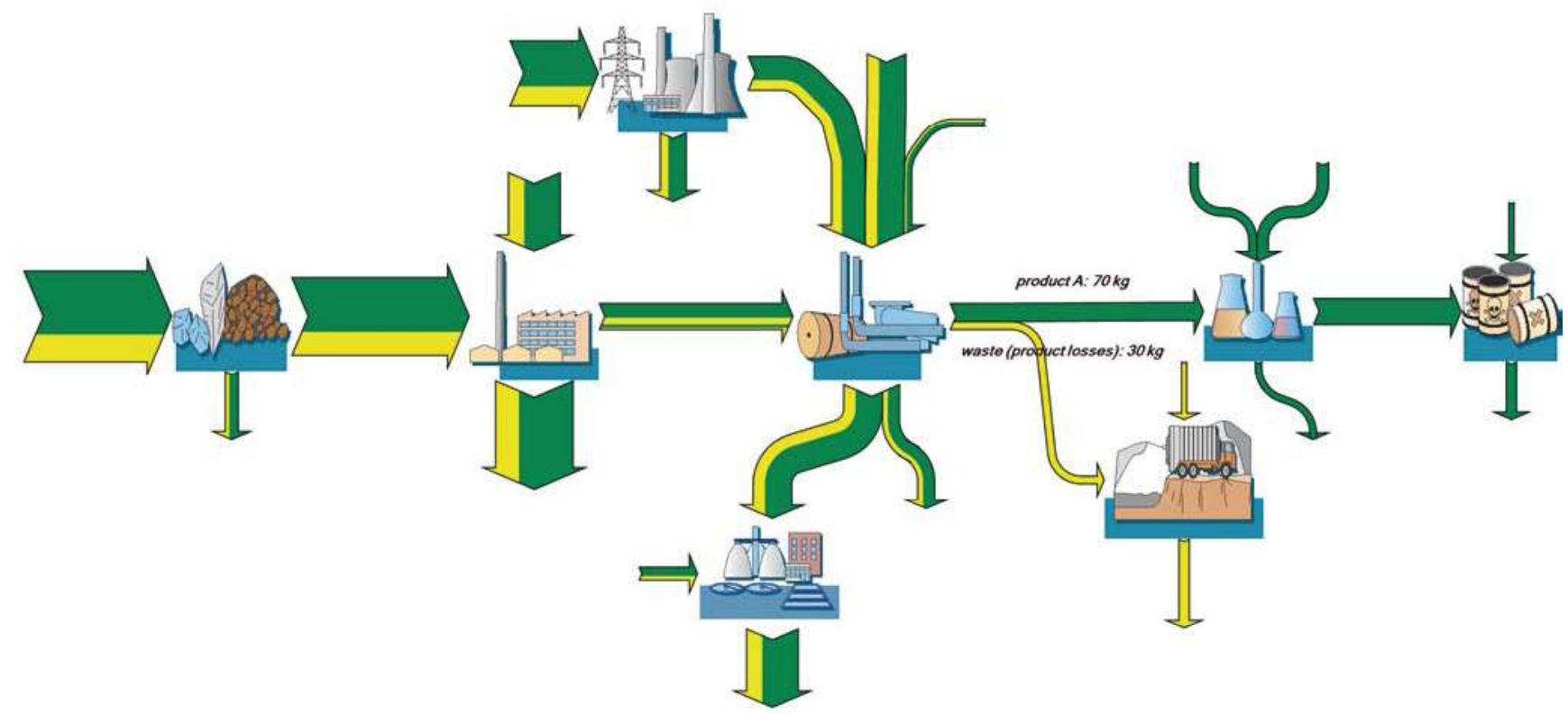

Abb. 3 Verschwendungs- und produktbezogene Flüsse in einem größeren Produktionszusammenhang. (Viere und Möller 2009)

ausweisen, die mit der Beseitigung von Ineffizienzen in der Produktion verbunden wäre.

\section{Export nach Japan}

In Japan wurde MFCA erstmals im Jahr 2000 verwendet. Von Professor Kokubu wurde ein Forschungsprojekt zur Umweltkostenrechnung geleitet, das vom Japanischen Ministerium für Industrie und Handel (METI) gefördert wurde. In diesem Zusammenhang besuchten Professor Nakajima und Professor Mizuguchi im Herbst 2000 das Institut für Management und Umwelt in Augsburg und führten mit Professor Wagner und Dr. Strobel ein Interview darüber, was MFCA ist, wie Flussmengen bestimmt und Materialflusskosten berechnet werden können. In der Folge wurde der MFCA-Ansatz in Japan näher untersucht und eine erste Fallstudie mit dem Unternehmen Nitto Denko wurde durchgeführt. Dies mündete in einen Bericht des Ministeriums, in dem die grundlegende Idee von MFCA und die erste Fallstudie Ende 2000 veröffentlicht wurden.

Das Projekt aus dem Jahr 2000 zeigte eine neue Perspektive zur Steigerung von Materialeffizienzen in Produktionsprozessen auf. Das Volumen der durch MFCA ermittelten Materialverluste war deutlich größer, als die Unternehmen erwartet hatten. Mehr als $30 \%$ Materialverluste, bezogen auf das Eingangsmaterial, war kein ungewöhnlicher Wert. Einige Unternehmen erkannten, dass die Reduktion der Materialverluste direkt zur Verminderung des Materialeinsatzes, damit zur Senkung der Produktionskosten beiträgt und zugleich ein wichtiges Element von Nachhaltigkeitsmanagement sein kann. Viele Unternehmen konnten bisher unerkannte Materialverluste in ihren Produktionsprozessen identifizieren. Einige Unternehmen haben durch MFCA große Vorteile erfahren (vgl. Nakajima \& Kokubu 2008).

Seither wurden bis zum aktuellen Jahr 2010 weitere METI-finanzierte Projekte fortgesetzt, um Methode und Anwendungskontext von MFCA weiterzuentwickeln. Die Anzahl der Unternehmen, die heute in Japan Erfahrungen mit MFCA gesammelt haben, liegt bei 200-300. Methodisch umfasst die Basis-MFCA in Japan zunächst die Analyse betrieblicher Produktionsmaterialien. Aber es gibt auch weiterreichende Ansätze, die etwa Informationen $\mathrm{zu} \mathrm{CO}_{2}$-Emissionen oder für ein Life Cycle Assessment zur Verfügung stellen. Die Basis-MFCA bezieht sich entweder auf einen bestimmten Herstellungsprozess oder auf eine ausgewählte Produktionslinie. Es werden derzeit aber auch in einem erweiterten Anwendungskontext Erfahrungen gesammelt, bei denen MFCA auf die Lieferkette ausgedehnt wird, somit also 2 oder 3 Unternehmen gemeinsam an einem MFCAProjekt arbeiten. Auf der anderen Seite wurde auch versucht, den Anwendungskontext von MFCA auf Forschung \& Entwicklung oder auf die Phase des Produktdesign auszudehnen (vgl. Nakajima 2010). Einige japanische Unternehmen haben MFCA auch in ihren Standorten außerhalb Japans eingeführt. Teilweise wurden die Dokumentation und die Fallstudien der japanischen Unternehmen auch schon auf Englisch übersetzt und stehen als gedruckte Exemplare oder per Website zur Verfügung. So nutzen auch schon einige Unternehmen in Korea, China oder einigen süd-ost-asiatischen Länder, MFCA. 


\section{Rückkehr als ISO-Norm 14051}

Vor diesem Hintergrund hat Japan in 2007 die Initiative ergriffen und die Erarbeitung einer grundlegenden Norm zu MFCA als neues Mitglied der ISO 14000er-Familie vorgeschlagen. Im Jahr 2010 hat dieser Standard mit der Bezeichnung ISO 14051 bereits den Status eines DIS (Draft of International Standard) im ISO Prozess erreicht. Im Juni 2010 wurde die erste deutsche Fassung des Entwurfs ISO 14051 Materialflusskostenrechnung - Allgemeine Rahmenbedingungen (ISO/DIS 14051 2010) entworfen. Während der bisherigen Diskussion über den Standard haben sich internationale Experten in die Überarbeitung und Ergänzung des Entwurfs eingebracht und Fallstudien aus verschiedenen Ländern und Branchen erarbeitet. Die grundlegende Idee zur Methode Materialflusskostenrechnung kam also von Deutschland nach Japan und gewinnt nun durch den ISO Prozess auch weltweit neue Anwender hinzu.

Die Struktur des Standards gliedert sich in folgende Elemente:

1. Anwendungsbereich

2. Begriffe

3. Zielsetzung und Grundsätze der $\mathrm{MFKR}^{1}$

4. Grundlegende Elemente der MFKR

5. Implementierungsschritte einer MFKR

Anhang A (informativ): Unterschied zwischen MFKR und konventioneller Kostenrechnung

Anhang B (informativ): Kostenrechnung und Zuordnung in der MFKR

Anhang C: Fallbeispiele einer MFKR

Die Intention der Norm ISO 14051 ist es, die generellen Prinzipien und Rahmenbedingungen für die Materialflusskostenrechnung darzustellen und $\mathrm{zu}$ standardisieren, um damit eine größere Verbreitung der Methode zu unterstützen und so weltweit zu einem effizienteren Umgang mit Ressourcen in Unternehmen beizutragen. Durch die Einfachheit im Grundkonzept und ihre Skalierbarkeit ist MFCA auch besonders für Vertreter oder Consultants kleinerer und mittlerer Unternehmen von Interesse, da die ersten Schritte ohne großen Aufwand umgesetzt werden können; dies zeigten diverse japanische Fallbeispiele (vgl. METI 2010).

Die Darstellung der grundlegenden Prinzipien und Elemente der Materialflusskostenrechnung erfolgt insbesondere in Kap. 3 und 4 der neuen Norm. Die vier grundlegenden Elemente, die zu jeder Materialflusskostenrechnung

\footnotetext{
${ }^{1}$ Als Abkürzung in der ersten deutschen Fassung des Standardentwurfs wird für Materialflusskostenrechnung „MFKR“ verwendet. Auch unter den deutschen Experten, die an der Standardentwicklung beteiligt sind, ist aber die englische Abkürzung „MFCA“ gebräuchlich. Die genauen Begriffe, die in der deutschen Fassung letztlich verwendet werden, können sich im Diskussionsprozess noch ändern.
}

gehören, sind: Mengenstelle (engl. Quantity Centre), Materialbilanz, Kostenrechnung und Materialflussmodell. Eine Mengestelle kann sich auf einen oder mehrere aggregierte Prozesse beziehen, für den Inputs und Outputs in physikalischen und monetären Einheiten erfasst werden (vgl. DIS ISO 14051, Deutsche Fassung 2010). Mengenstellen sollen vor allem Orte von Materialverlusten genauer identifizieren und der Prozesssteuerung zugänglich machen. Detaillierte Vorgehensweisen für die Berechnung der Materialflusskosten werden nicht durch den Standard abgedeckt, vielmehr werden die grundlegenden Schritte, die für eine Implementierung erforderlich sind, einführend dargestellt. Die Beschreibung der Implementierungsschritte in der Norm stellt eine Anleitung für jedes Unternehmen dar, das am Anfang der Einführung von MFCA steht.

Es handelt sich bei ISO 14051 nicht um einen zertifizierbaren Standard, da die Methode MFCA auf interne Belange von Unternehmen fokussiert ist. Der Standard ist als komplementär und ergänzend zu bereits existierenden Standards der ISO 14000er Familie zu Umweltmanagementsystemen, Ökobilanzierung, Umweltleistungsbewertung oder auch zu Green House Gas Accounting anzusehen. In Bezug auf Umweltmanagementsysteme, kann die neue MFCA-Norm besonders gut Informationen für den Kontinuierlichen Verbesserungsprozess liefern und immer wieder Verbesserungspotenziale aufdecken. Mit der Ökobilanzierung hat MFCA das Material- und Energieflussmodell als Basis gemeinsam, wobei die Ökobilanz grundsätzlich eine Lebenszyklusperspektive einnimmt und die Bewertung der Umweltwirkungen eines Produktes zum Ziel hat. MFCA ergänzt diesen Ansatz insbesondere um ökonomische Aspekte.

Weiterhin basiert die Ermittlung und Bewertung der $\mathrm{CO}_{2}$ Emissionen im Rahmen des Managements klimarelevanter Gase in der Regel auf einer Auswertung der Eingangsmaterialien, die als Energieträger fungieren, hier stellt die regelmäßige Erfassung der Material- und Energieströme für die Produktionsprozesse im Rahmen von MFCA eine deutliche Verknüpfung dar (vgl. Kokubu et al. 2009).

Der Standard ISO 14051 wird derzeit in der Arbeitsgruppe 8 des ISO-Gremiums TC207 bearbeitet (ISO/TC 207/WG 8). Diese setzt sich aus ca. 50 internationalen Experten aus 24 Ländern zusammen. Die Arbeitsgruppe hat sich bislang zu vier Arbeitstreffen in Bogotá (Kolumbien), Tokio (Japan), Kairo (Ägypten) und Prag (Tschechien) getroffen. Im Rahmen aller Treffen fand im Vorfeld auch ein öffentlicher Workshop mit Vorträgen statt, in denen sowohl die Methodik als auch Fallstudien aus unterschiedlichen Ländern vorgestellt wurden. Im Rahmen des letzten TC207Jahrestreffens, bei dem viele Arbeitsgruppen der 14000er Familie zusammenkommen, fand im Juli 2010 erneut ein Workshop statt, auf dem erstmals auch MFCA-Fallstudien aus Malaysia und Mexiko vorgestellt wurden. 


\section{Ausblick}

In Japan zeichnet sich eine weitere dynamische Entwicklung von MFCA als etabliertes Tool des Nachhaltigkeitsmanagements ab, das insbesondere die Zielsetzsung der Prozessinnovation verfolgt. MFCA wird weiterhin im Supply Chain Management, im Management von $\mathrm{CO}_{2}$-Emissionen, sowie in Life Cycle-Projekten und ähnlichen Kontexten erprobt und weiterentwickelt. Zukünftig soll MFCA in Japan dahingehend entwickelt werden, dass die Methode auch einen Beitrag zur sozialen Nachhaltigkeit leisten kann.

In Deutschland und Europa steht das Thema stärker hinsichtlich der Ressourceneffizienz im Fokus von Politik und Unternehmen. Mit der Veröffentlichung des Standards ISO 14051 wird den betrieblichen Materialverlusten und Ineffizienzen in der Produktion eine verstärkte Aufmerksamkeit zukommen. Die Veröffentlichung des finalen Standards ist nach ISO-Prozeduren für 2012 geplant.

\section{Literatur}

Berger M et al (2003) Flussmanagement für Produktionsunternehmen. Material- und Informationsflüsse nachhaltig gestalten. imu Augsburg GmbH \& Co. KG und Zentrum für Weiterbildung und Wissenstransfer, Augsburg

Brunner PH, Rechberger H (2003) Practical handbook of material flow analysis. CRC, Boca Raton

DIN EN ISO 14051 (2010) Umweltmanagement - Materialflusskostenrechnung - Allgemeine Rahmenbedingungen; Deutsche Fassung prEN ISO 14051:2010. Beuth, Berlin

Hessisches Ministerium für Wirtschaft, Verkehr und Landesentwicklung (Hrsg) (1999) Leitfaden „Flusskostenmanagement - Kostensenkung und Öko-Effizienz durch eine Materialflussorientierung in der Kostenrechnung“. Hessisches Ministerium für Wirtschaft, Verkehr und Landesentwicklung, Wiesbaden, S 72

Jasch C (2009) Environmental and material flow cost accounting: principles and procedures. Springer, Dordrecht
Kokubu K, Campos MKS, Furukawa Y, Tachikawa H (2009) Material flow cost accounting with ISO 14051. ISO Management Systems (January-February 2009), S 15-18

Landesanstalt für Umwelt Baden-Württemberg (LfU) (Hrsg) (1999) Leitfaden „Betriebliches Material- und Energieflussmanagement - Öko-Effizienz durch nachhaltige Reorganisation". Landesanstalt für Umwelt Baden-Württemberg (LfU), Karlsruhe, S 35

Letmathe P, Stürznickel B, Tschesche J (2002) Ressourcenkostenrechnung. Umweltwirtschaftsforum 2002(10):52-57

Loew T, Fichter K, Müller U (2003) Ansätze der Umweltkostenrechnung im Vergleich. Umweltbundesamt UBA-Texte 78/03, Berlin

METI - Japanese Ministry of Economy, Trade and Industry (2007) Guide for material flow cost accounting. METI, Tokyo

METI - Japanese Ministry of Economy, Trade and Industry (2010) Material flow cost accounting mfca case examples. METI, Tokyo

Nakajima M (2010) Environmental management accounting for sustainable manufacturing: establishing management system of Material Flow Cost Accounting (MFCA). Kansai Univ Rev Bus Commer 2010(12):41-58 (in English)

Nakajima M, Kokubu K (2008) Material flow cost accounting, 2. Aufl. Nihonkeizai-shinbun-sya (1. Aufl. 2001, in Japanese)

Schaltegger S, Burritt RL (2000) Contemporary environmental accounting - issues, concepts and practice. Greenleaf, Sheffield

Strobel M, Redmann C (2002) Flow cost accounting, an accounting approach based on the actual flows of materials. In: Bennett M, Bouma JJ, Wolters T (Hrsg) Environmental management accounting: informational and institutional developments. Springer, Dordrecht, S 67-82

Viere T, Möller A (2009) A material flow cost accounting approach to improvement assessment in LCA, Tagungsband. 3rd International Seminar on Society \& Material, Freiberg

Wagner B, Enzler S (Hrsg) (2006) Material flow management: improving cost efficiency and environmental performance. Physica, Heidelberg

Wagner B, Rauberger R (1999) Ecobalance analysis as a managerial tool at Kunert AG. In: Bennett M, James P (Hrsg) Sustainable measures. Greenleaf, Sheffield, S 170-184

Wagner B, Strobel M (1999) Kostenmanagement mit der Flusskostenrechnung. In: Jürgen Freimann (Hrsg) Werkzeuge erfolgreichen Umweltmanagements. Gabler, Wiesbaden, S 49-70 